\title{
Fabrication of poly- and single-crystalline platinum nanostructures using hole-mask colloidal lithography, electrodeposition and annealing
}

\author{
Björn Wickman, Hans Fredriksson ${ }^{1}$, Stefan Gustafsson, \\ Eva Olsson and Bengt Kasemo \\ Department of Applied Physics, Chalmers University of Technology, SE-412 96 Göteborg, \\ Sweden \\ E-mail: bjorn.wickman@chalmers.se and kasemo@chalmers.se
}

Received 18 May 2011, in final form 13 July 2011

Published 3 August 2011

Online at stacks.iop.org/Nano/22/345302

\begin{abstract}
Colloidal lithography (CL) is a generic name for a collection of nanolithographic techniques, based on using colloidal nanoparticles as pattern (mask)-defining entities to produce various nanostructures. A key step in CL processes is the deposition, usually by evaporation or sputtering, of the material that makes up the final nanostructures. We have for the first time combined a special version of CL, called hole-mask colloidal lithography (HCL), with electrodeposition. We demonstrate how electrodeposition of Pt onto Au and carbon substrates, through a lithographic mask, can be used to prepare well-defined nanostructured surfaces. The results are compared with evaporated structures and characterized by scanning electron microscopy (SEM), transmission electron microscopy (TEM) and cyclic voltammetry. Specific results are: (i) electrodeposition generates structures with very good adhesion, (ii) due to differences in the deposition mechanism, structures with much larger aspect (height/width) ratio can be made with electrodeposition than with evaporation and (iii) the originally deposited polycrystalline nanoparticles can be annealed into single crystals, as demonstrated by electron diffraction, SEM and TEM, before and after annealing, which is of great value for fundamental (electro)catalysis studies.
\end{abstract}

\section{Introduction}

Platinum is one of the most commonly used catalytic materials in general catalysis and in applications such as catalytic converters and low temperature fuel cells, and is also one of the most common catalysts in fundamental model studies. Fabrication of well-defined, and systematically varied, nanostructured model systems of supported Pt is therefore of both practical and generic importance and has been one of the reasons for the development of a field called nanocatalysis. During the past few decades there has been a rapid development of methods to fabricate various nanocatalysis model systems [1, 2]. They range from

1 Present address: Department of Chemical Engineering and Chemistry, Eindhoven University of Technology, 5600 MB Eindhoven, The Netherlands. very expensive and demanding methods like electron beam lithography (EBL) [3] to less precise, but faster and cheaper methods, like those based on self-assembly [4]. There is, however, still a need for additional methods, which are simultaneously versatile and precise, and able to produce samples with simpler and lower cost equipment.

An example moving in the latter direction is the family of lithographic bottom-up techniques to fabricate 2D nanostructured surfaces, using templates formed from selfassembly of colloidal particles, often collectively termed colloidal lithography (CL) $[5,6]$. Depending on the conditions applied during deposition of the nanospheres, either close packed or sparse layers with well-defined inter-particle distances can be achieved [7,8]. The colloidal particles can function either as an evaporation mask or an etch mask 
to fabricate nanostructures with a variety of shapes with well-defined sizes (typically from a few tens of nanometers and up) and separations, in essentially any material [7, 9-11].

Our group has recently developed a new CL technique, termed hole-mask colloidal lithography (HCL) [12], which has rapidly come into broad use in fabrication of large-area samples (several $\mathrm{cm}^{2}$ ) for exploring, for example, fuel-cellrelated electrochemistry, gas phase catalytic processes and localized surface plasmon resonance sensing systems [13-17]. The method employs a sacrificial layer (usually a thin polymer film) and a sparse (far from close packed) layer of charged colloidal particles to define an evaporation mask. This enables fabrication of several types of particle shapes, such as ellipses, cones and proximal particle pairs with variable interparticle distance, which cannot be fabricated by the standard methods [12].

A crucial step in most lithographic methods is the deposition of the material(s) that will constitute the nanostructures. This is often done by physical vapor deposition in vacuum (PVD; evaporation or sputtering), which has both advantages, like clean conditions and the possibility to vacuum-anneal the structures, as well as drawbacks. Among the latter is a very low material utilization, since the majority of the consumed material is deposited on areas away from the sample (i.e. on the deposition chamber walls, shutters, screens or on the evaporation mask). For expensive materials, such as $\mathrm{Pt}$, this increases the fabrication cost significantly. Moreover, the evaporation must be carried out in high quality vacuum systems, with very pure source materials and clean substrates to ensure contamination-free and stable samples [18]. Finally, other deposition methods, like the electrodeposition described here, can, on some substrates and/or for some materials, offer advantages also with regard to nucleation and growth, influencing the microstructure and/or adhesion, and, as demonstrated below, also with regard to the aspect ratios of fabricated particles.

The results reported in this work extend the application range of the HCL technique, making it much more versatile and, in particular, eliminates or reduces some of the drawbacks mentioned above. Several novel modifications of the process are presented. As specific demonstrators we have chosen the following examples; (i) electrodeposition of Pt nanoparticles through an HCL mask onto $\mathrm{Au}$ and carbon substrates, (ii) annealing of deposited polycrystalline Pt nanoparticles to form arrays of single-crystal nanoparticles and (iii) detailed transmission electron microscopy (TEM) analysis of very small annealed Pt particles.

\section{Experimental details}

\subsection{Substrate preparation}

Si wafers (Siltronix), cut into $1 \mathrm{~cm}^{2}$ pieces, were used as substrates for the electrodeposition experiments (example (i) above). The $\mathrm{Si}$ was pre-covered with a thin $\mathrm{Ti}$ adhesive layer, followed by evaporation of $40 \mathrm{~nm} \mathrm{Au}$ or $50 \mathrm{~nm} \mathrm{C}$ functioning as active support material. For the detailed TEM analysis of small annealed $\mathrm{Pt}$ structures (example
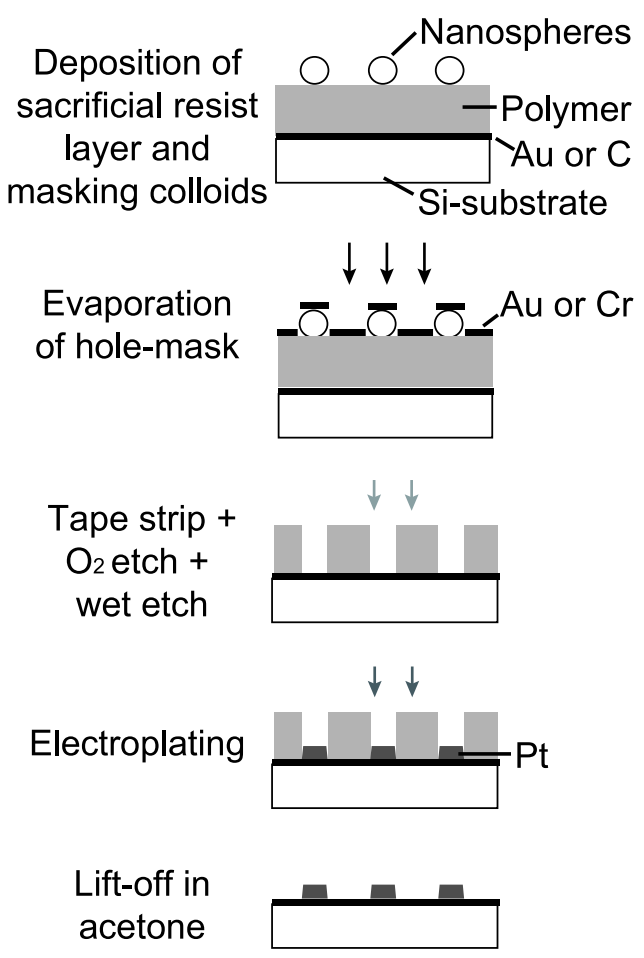

Figure 1. Schematic illustration of the fabrication processes used for the electrodeposited Pt nanostructures.

(iii) above), thin electron-transparent $\mathrm{Si}$-supported $\mathrm{Si}_{3} \mathrm{~N}_{4}$ membranes (TEM windows) were used as substrates to facilitate TEM imaging [19]. The membranes are very fragile and therefore a thick layer of polymer (PMMA) was deposited on the back of the TEM window to stabilize it and prevent it from breaking during fabrication. The polymer was removed by acetone before TEM imaging.

\subsection{Mask preparation with $H C L$}

The fundamental fabrication steps of preparing the lithographic mask with HCL largely followed the route outlined in [12]. The basic steps used in this work for the combination with electrochemistry are shown schematically in figure 1. Firstly, a sacrificial polymer film, poly(methyl methacrylate) (PMMA), $4 \%$, diluted in anisole, was spin-coated onto the surfaces for $1 \mathrm{~min}$ at $2000 \mathrm{rpm}$, baked on a hotplate for $10 \mathrm{~min}, 170^{\circ} \mathrm{C}$, and activated in an oxygen plasma $(5 \mathrm{~s}, 50 \mathrm{~W}, 250 \mathrm{mTorr}$, Plasma Therm batchtop RIE $95 \mathrm{~m}$ ). The PMMA surfaces were then provided a net positive charge by pipetting a polyelectrolyte onto the film (polydiallyldimethylammonium MW 200000-350000, Sigma Aldrich, 0.2 wt\% in Milli-Q water, Millipore), followed by rinsing with deionized water and blow-drying in $\mathrm{N}_{2} .190$ or $110 \mathrm{~nm}$ in diameter, negatively charged polystyrene particles (PS, sulfate latex, Interfacial Dynamics Corporation) were deposited in a similar fashion as described above, leaving the PMMA surface covered with uniformly distributed PS spheres. An oxygen-plasma-resistant film, which later functioned as the hole-mask, was evaporated onto the nanospheres + polymer film. This film consisted of a $20 \mathrm{~nm} \mathrm{Cr} / 10 \mathrm{~nm} \mathrm{Au}$ for the samples on Au-covered 
$\mathrm{Si}$ and a $20 \mathrm{~nm} \mathrm{Au}$ film for the samples on carbon-covered $\mathrm{Si}$. The PS spheres were removed by tape stripping (SWT10 tape, Nitto Scandinavia AB), leaving the metal hole-mask on the samples with the polymer film exposed in the holes. Oxygen plasma was used to etch the exposed polymer (i.e. the polymer not protected by the remaining gold film) down to the substrate. The hole-mask was removed by selective etching in Au etch (Gold etch 22 196, Sunchem Electrograde Products) for the carbon-covered samples, or Cr-etch (nickel-chrome etch, 711.21, Sunchem Electrograde Products) for those where an Au film was initially deposited onto the Si wafers. This procedure leaves only the structured polymer film mask on the surface. The reason for this was to avoid deposition on the metal hole-mask in the subsequent electroplating. No indications that this wet etch affected the substrate or the following process was found. $\mathrm{Pt}$ was then electroplated through the polymer mask, onto the Au or $\mathrm{C}$ substrates at the bottom of the holes. The polymer mask was finally dissolved in acetone, leaving only the disc-shaped nanostructures on the surfaces.

The fabrication of the samples prepared on TEM windows largely followed the same procedure as described above, but with a few important differences. Here, the nanospheres + polymer film was exposed to a short oxygen plasma prior to deposition of the hole-mask, effectively reducing the size of the nanospheres $[7,20]$ and also etched the polymer not immediately covered by the nanospheres. The hole-mask was then deposited by evaporation of $20 \mathrm{~nm}$ $\mathrm{Au}$ from two opposite angles, $45^{\circ}$ from the surface normal, resulting in holes smaller than the diameter of the original nanospheres. For these samples, $10 \mathrm{~nm}$ of Pt was evaporated through the Au mask to create nanoparticles on the support.

\subsection{Electrodeposition and electrochemical characterization}

A standard signal cable was attached to the samples by conductive Ag glue, subsequently shielded with nail varnish to ensure isolation. A potentiostat (Gamry REF600) was used to perform the electroplating and the cyclic voltammetry (CV) measurements. The experiments were conducted at room temperature in a standard three-electrode cell with a Pt wire as the counter electrode and an $\mathrm{Ag} / \mathrm{AgCl}$ reference electrode (Schott, B3420). However, all potentials in this report are expressed versus the reversible hydrogen electrode (RHE). The electrolyte was $0.5 \mathrm{M} \mathrm{H}_{2} \mathrm{SO}_{4}$ (Ficher Scientific and Milli-Q water) bubbled with $\mathrm{N}_{2}$. For the electroplating experiments, $\mathrm{H}_{2} \mathrm{PtCl}_{6}$ (Alfa Aesar) was added to the electrolyte to yield a Pt concentration of $0.5 \mathrm{mM}$. On the Au substrates, electroplating was carried out under chronopotentiostatic conditions at a current of $0.55 \mathrm{~mA} \mathrm{~cm}^{-2}$. On the carbon substrates, electroplating was performed using repeated potential pulsing where every pulse consisted of $0.1 \mathrm{~s}$ at $-0.3 \mathrm{~V}$ followed by $1 \mathrm{~s}$ at open circuit potential. $\mathrm{CV}$ curves were recorded between $0.025-1.4 \mathrm{~V}$ with a scan rate of $100 \mathrm{mV} \mathrm{s}^{-1}$.

\subsection{Physical deposition and annealing}

Deposition of the active support films of $\mathrm{Au}$ and $\mathrm{C}$ on $\mathrm{Si}$ and the Pt particles on TEM windows was carried out using e-gun evaporation (AVAC HVC 600). The Au and Cr hole-masks were deposited by resistive evaporation (Edwards 306 Evaporator). The electroplated $\mathrm{Pt}$ nanoparticles on $\mathrm{C}$ were heat-treated in a home-built quartz tube flow reactor in $4 \% \mathrm{H}_{2}$ in Ar flowing over the sample at $1023 \mathrm{~K}$ for $10 \mathrm{~min}$. The Pt nanostructures on TEM windows were heat-treated in a rapid thermal processor (RTP, JIPELEC Jet First 200) in an Ar atmosphere at $1023 \mathrm{~K}$ for $3 \mathrm{~min}$.

\subsection{Electron microscopy}

Scanning electron microscopy (SEM) images of the samples were acquired using a Zeiss Supra 60 operating at $10 \mathrm{kV}$ in the secondary electron mode. Transmission electron microscopy (TEM) images were acquired using a Philips CM200 operating at $200 \mathrm{kV}$.

\section{Results and discussion}

\subsection{Pt electroplating on Au substrates}

Figure 2 shows SEM images of electroplated Pt nanodisc arrays deposited on Au supports, prepared as described above. A constant current density of $0.55 \mathrm{~mA} \mathrm{~cm}^{-2}$ was used for three different deposition durations, 8 (figure 2(a)), 30 (figure 2(b)) or $180 \mathrm{~s}$ (figure 2(c)). Based on the charges passed during the experiment, the (average) thicknesses of the deposited Pt were estimated to be 7, 26 and $155 \mathrm{~nm}$, respectively. Au substrates are uniform and excellent conductors and thus the Pt grows homogeneously, creating flat nanodiscs with an almost perfect circular perimeter. The top of the thicker nanodiscs shows a larger relative roughness than the thinner ones, in accordance with previous findings [21].

\subsection{Pt electroplating on $C$ substrates}

In practice it is essential that the electroplating can be applied also on support materials which have lower or less homogeneous conductivity than $\mathrm{Au}$ (of course, the support must have a reasonable electrical conductivity to be used at all with electrodeposition). Examples of such supports are amorphous carbon and polycrystalline graphite, which are often used in low temperature fuel cell, solar cell, and battery related studies. Electroplating on these substrates often results in non-homogeneous film growth $[22,23]$. In the case of plating through a lithography mask, the limited conductivity could potentially result in material being deposited only in a fraction of the holes or with uneven size/thickness. This was indeed also found in this work for chronopotentiostatic deposition on carbon (results not shown).

To minimize these effects, repeated potential pulse deposition was employed for electrodeposition of Pt on carbon. Figure 3 shows SEM images of electroplated Pt nanodisc arrays, pulse-deposited on $\mathrm{C}$ supports. The samples in figure 3 were deposited with 25 (figure 3(a)) or 200 pulses (figure 3(b)). After 25 pulses, there was at least one small Pt particle formed in every hole in the polymer mask. The electrodeposited Pt did, however, not yet completely cover the exposed carbon surface, but instead Pt hemispheres of about $50 \mathrm{~nm}$ were formed, most 

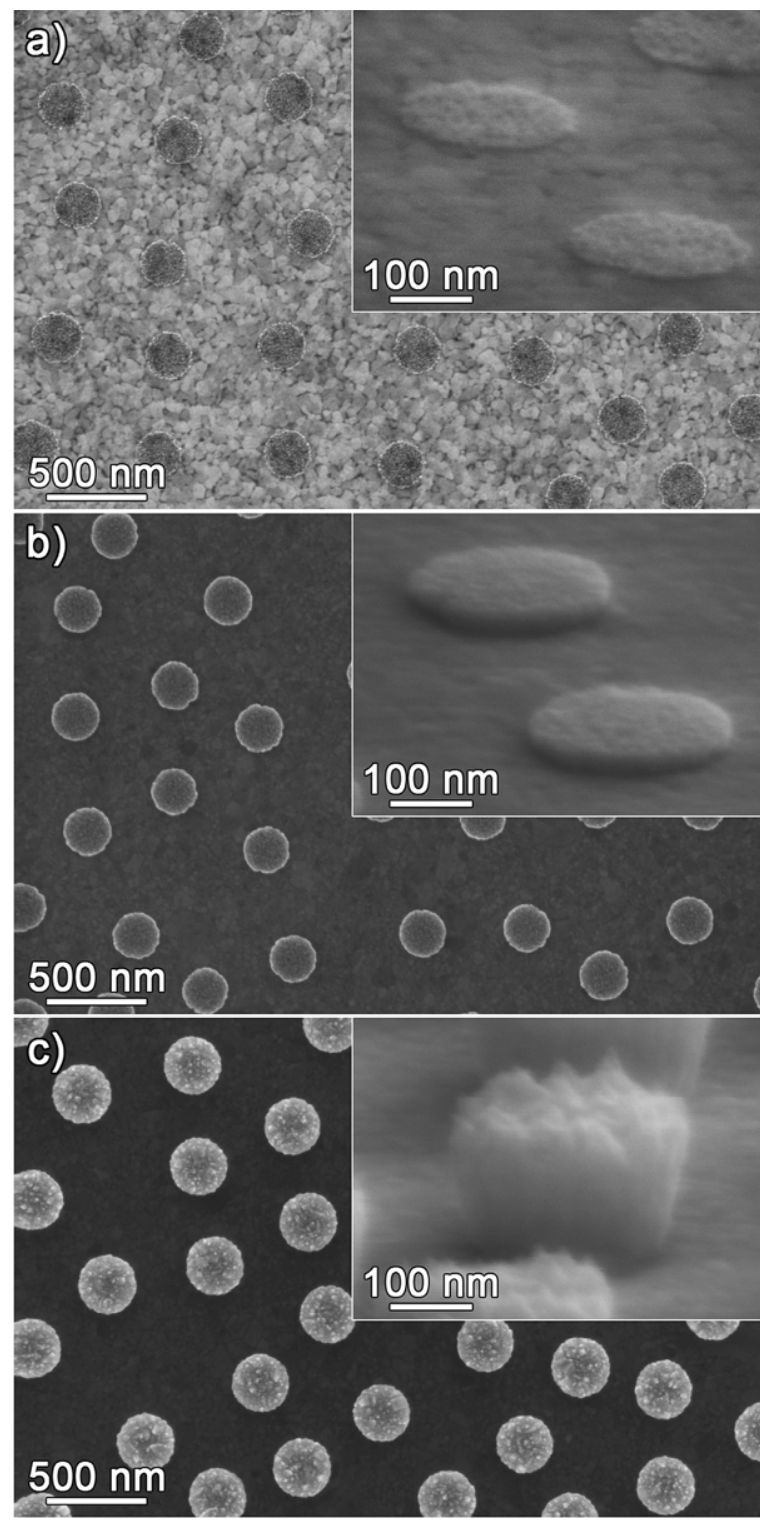

Figure 2. SEM images of Pt nanoparticles electrodeposited on $\mathrm{Au}$ support at $0.55 \mathrm{~mA} \mathrm{~cm}^{-2}$ for $8 \mathrm{~s}$ (a), $30 \mathrm{~s}$ (b) and $180 \mathrm{~s}$ (c). Tilted, high magnification SEM images are shown as insets.

likely on sites that favored nucleation [23]. Moreover, a few very large (up to about $1 \mu \mathrm{m}$ ) particles were formed as well. These could effectively be removed by sonication at full power in an ultrasonic bath, while the smaller Pt structures were not influenced at all by this rather violent treatment, suggesting good adhesion to the substrate. For the 200 pulse sample, the deposition was divided into four sequential sets of 50 pulses, with sonication in between. After this, the exposed carbon surface in all the holes of the mask had been completely covered, rendering a full layer of Pt nanodiscs. Although the homogeneity of the produced nanostructures was not as excellent as for those deposited on the Au substrates, it was judged satisfactory for the subsequent experiments. Optimization of the deposition is likely to be achieved by, for example, varying the potentials, pulse durations and the number of pulses. However, the present results already show
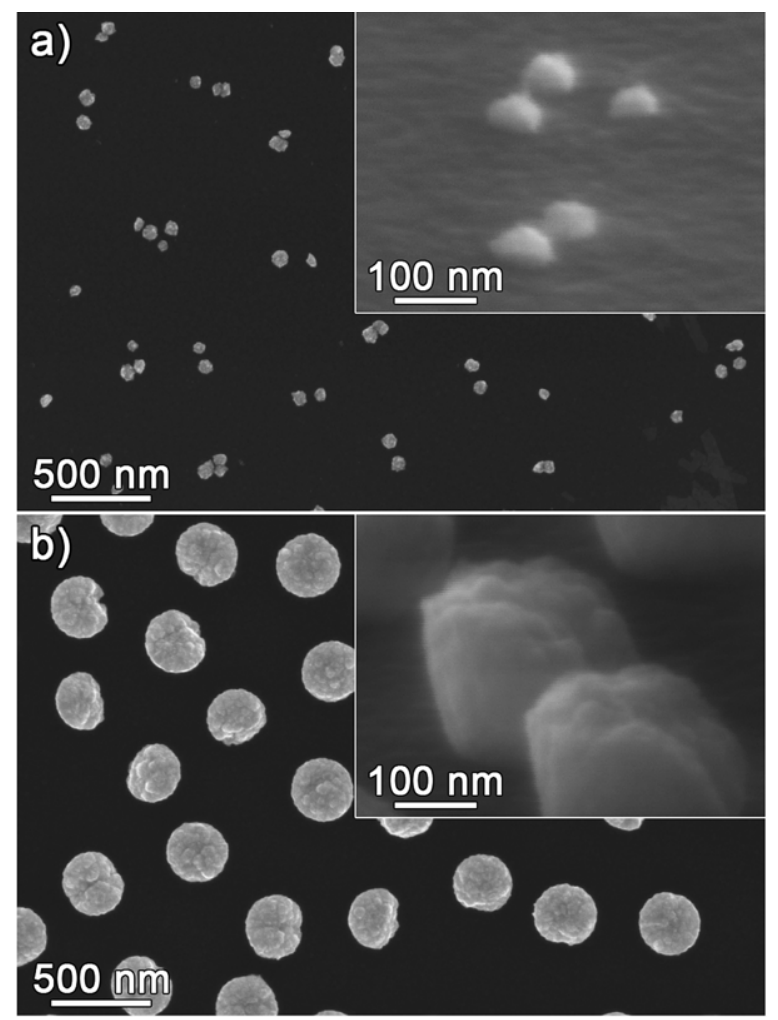

Figure 3. SEM images of Pt nanoparticles electrodeposited on carbon support with 25 pulses (a) and 200 pulses (b). Tilted, high magnification SEM images are shown as insets.

that it is possible to electrodeposit well-defined nanoparticles of Pt also on an amorphous carbon support.

\subsection{Electrochemical characterization}

To examine the electrochemical properties of the fabricated structures, one sample of $\mathrm{Pt}$ plated on $\mathrm{Au}$ (30 s plating, figure 2(b)) and one on carbon (200 pulses, figure 3(b)) were analyzed by cyclic voltammetry (CV). The results are shown in figure 4, where a reference sample consisting of a $20 \mathrm{~nm}$ evaporated Pt film was also included for comparison. The quoted current densities are based on the total exposed sample surface (projected area). All samples display voltammograms typical of clean $\mathrm{Pt}$, indicating that the surfaces were free from contamination affecting the $\mathrm{CV}$ properties. The Pt discs on $\mathrm{Au}$ had an electrochemical surface area (ECSA) of Pt (based on hydrogen underpotential deposition charge [24]) that was about $40 \%$ of that of the continuous Pt film. Comparing the ECSA with the geometric surface area of the nanodiscs (estimated from the SEM images) yielded a roughness factor (RF) of about 4.5, which indicates that the electroplated Pt has a larger nanoroughness than the evaporated film, or possibly even a nanoporous structure. The RF for the evaporated Pt film was about 2.5 , in good agreement with previous reports [25]. It can be noted that this RF value also implies a relatively high roughness. For the Pt discs on carbon, the ECSA was about 1.6 times larger than that of the Pt film, which can be explained by the fact that the Pt particles were rather high (about $200 \mathrm{~nm}$ ) and had a rough top surface, as seen in figure 3(b). 


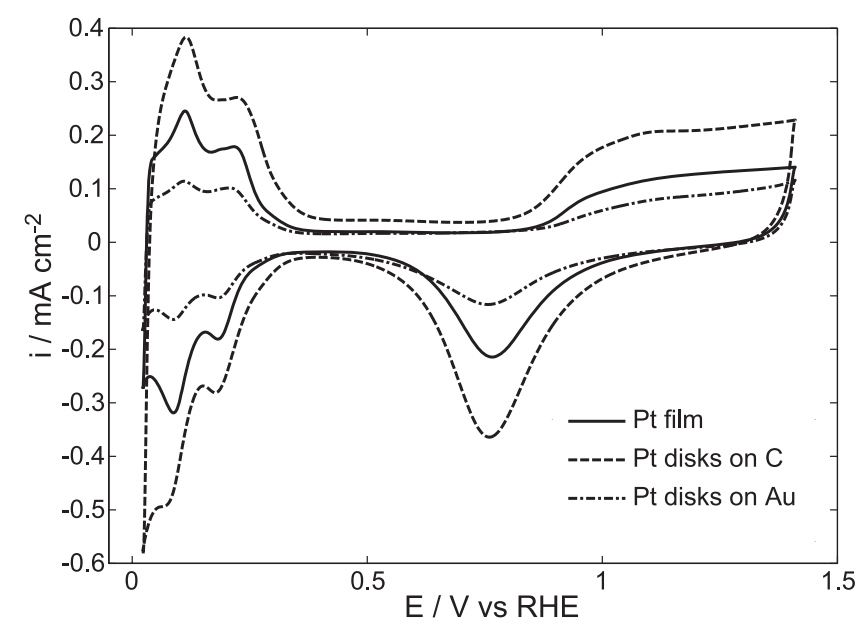

Figure 4. Cyclic voltammetry curves measured in $\mathrm{N}_{2}$-bubbled $0.5 \mathrm{M}$ $\mathrm{H}_{2} \mathrm{SO}_{4}$ for the 30 s electroplated sample on $\mathrm{Au}$ (dashed-dotted) (sample shown in figure 2(b)), the 200 pulse sample on carbon (dashed) (sample shown in figure 3(b)) and an evaporated Pt film (solid). A scan rate of $100 \mathrm{mV} \mathrm{s}^{-1}$ was used and the current density was based on the projected area of the samples.

The electrochemical stability of the samples was evaluated by cycling the potential between $0.025-1.4 \mathrm{~V}$ for more than 100 cycles, after which no decrease in the ECSA was observed. This, and the fact that no particle loss was observed after the ultrasonic treatment, prove that the samples have high stability and adhesion, and that they are suitable as electrodes for electrochemical studies.

It has previously been shown that electrodeposition can be combined with lithography. For example, arrays of $\mathrm{Ni}$ nanoparticles deposited through an EBL mask [26], arrays of Pt nanodots from a template derived from close-packed colloidal spheres [27] and 3D macroporous films of different metals and metal oxides have been described [28-30]. Replacing evaporation as the method of depositing the active material in lithography processes with electrodeposition has several advantages. Electroplating equipment is, in general, much cheaper and requires less maintenance than PVD deposition equipment and material deposition is restricted to the areas of interest (i.e. the unmasked areas of the sample). The material utilization is therefore $100 \%$ for electroplating as compared to less than $0.1 \%$ for a typical evaporation system. Electrodeposition can also be used to deposit materials that cannot readily be produced by evaporation, for example, alloys [31]. There are additional advantages when electroplating is combined with HCL. Since the height of the sacrificial layer can be controlled (and be much higher than the diameter of the colloids) and the holes etched through the polymer layer are cylindrical, the nanoparticles will be disc- or pillar-shaped rather than the cup-shaped particles or networks of materials that are obtained when electroplating is combined with conventional CL techniques. Furthermore, when material is evaporated though an HCL or EBL mask, material will stick to the sides of the mask and eventually close the holes, rendering cone-shaped nanoparticles. This will not happen during electroplating and thus it is possible to create

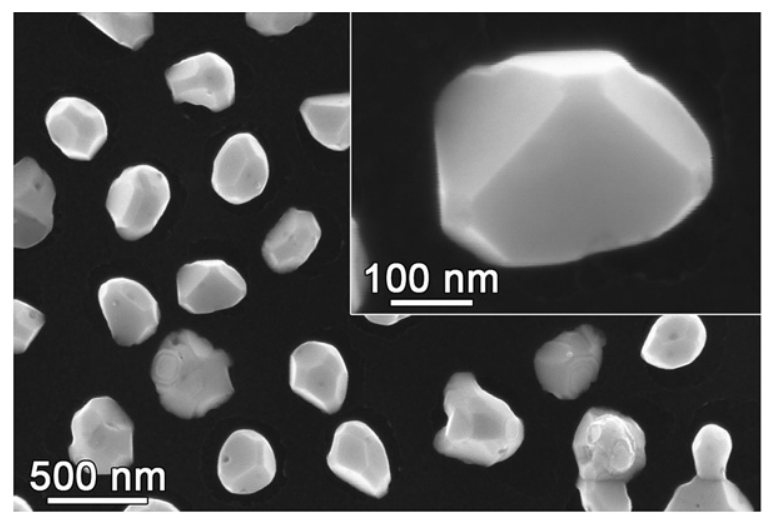

Figure 5. SEM image of the sample shown in figure 3(b) after annealing. A high magnification image is shown as the inset.

nanostructures with higher aspect (height/diameter) ratios than with evaporation.

\subsection{Annealing of Pt nanodisc arrays}

A common feature of $\mathrm{Pt}$ films formed in non-equilibrium processes (e.g. electrodeposition or evaporation) is that they are polycrystalline. This is a consequence of the many initial nucleation points on the surface at the beginning of the deposition, and also due to the later growth stage, where atoms may be stuck in other than lowest energy sites. In each hole in the HCL mask, the initial nuclei will eventually grow together and coalesce into a single nanoparticle. Such polycrystalline nanoparticles exhibit lattice compression, microstrains and intergrain boundaries. The amounts of defects and the relative fractions of surface to bulk atoms depend on parameters like the deposition technique, support material, deposition rate and deposition temperature [21]. The non-equilibrium state of the crystallites will also infer a high roughness (e.g. the high ECSA of both evaporated and electroplated Pt shown in figure 4). These differences will, in turn, affect the catalytic behavior of the material and can complicate comparisons between differently prepared samples [32-34]. By proper post-deposition annealing these differences and defects can be reduced or eliminated by forming single-crystal nanoparticles.

Figure 5 shows SEM images of the electrodeposited $\mathrm{Pt}$ structures shown in figure 3(b) after annealing in a gas flow reactor at $1023 \mathrm{~K}$ in $4 \% \mathrm{H}_{2}$ in Ar flowing over the sample. The $\mathrm{H}_{2}$ was added in order to react any $\mathrm{O}_{2}$ impurities that would otherwise oxidize the carbon support. As can be seen in the low magnification overview, the shape of the $\mathrm{Pt}$ nanoparticles changed significantly during the annealing and most particles display well-defined facets. However, there is a large spread in particle and facet shape which is likely to be a consequence of the fact that the particles had irregular shapes from the fabrication (figure 3(b)) and that the annealing was not optimized to allow all particles to reach equilibrium shape. Nonetheless, a few particles displayed very regular shapes, likely to be the Wulff shape, and an example can be seen in the inset of figure 5. This particle is predominantly terminated by (111) planes, with a small fraction of (100) facets. It is likely that the amount of regular particles can be increased by 

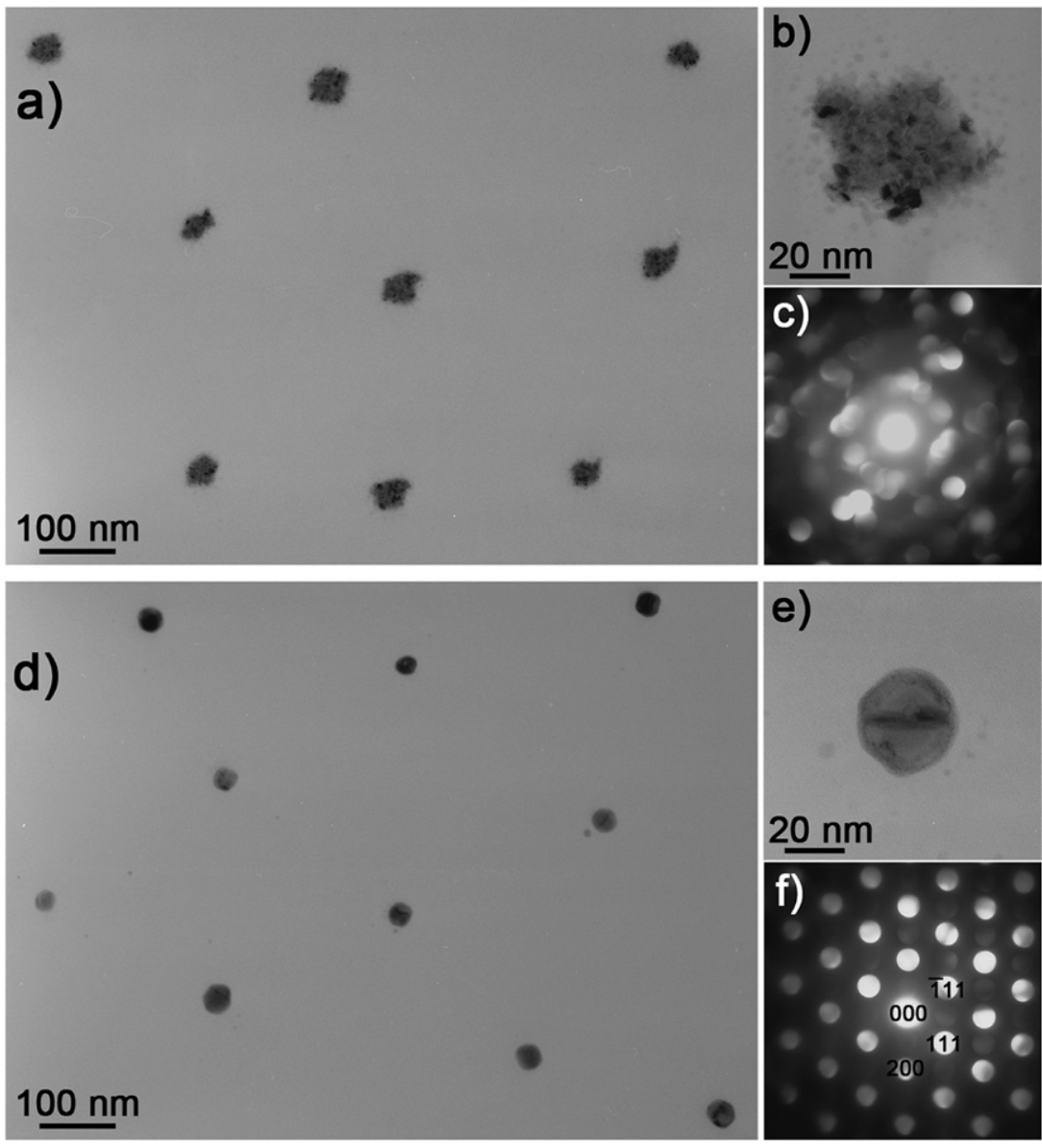

Figure 6. TEM images of Pt nanoparticles before (overview (a), high magnification (b)) and after annealing at $1023 \mathrm{~K}$ in Ar (overview (d), high magnification (e)) together with CBED patterns from individual particles before (c) and after annealing (f).

improving the fabrication and optimization of the annealing procedure.

To examine the effect of annealing in greater detail, samples of smaller Pt particles deposited on TEM windows were prepared. Figures 6(a) and (b) show TEM images of the as-prepared $\mathrm{Pt}$ structures deposited by vapor deposition. Nanospheres with a diameter of $110 \mathrm{~nm}$ were used in the fabrication, while the diameter of the produced $\mathrm{Pt}$ nanoparticles was found to be about $45 \mathrm{~nm}$. As a consequence, the particle coverage was about $1.5 \%$, compared to about $10 \%$ for the original nanospheres, but with preserved spatial order, i.e. without changing center-of-gravity particle positions. The Pt particles are polycrystalline, which is seen in the convergent beam electron diffraction (CBED) pattern from an individual particle, shown in figure 6(c). The sample shown in figures 6(a)-(c) was annealed in Ar atmosphere at $1023 \mathrm{~K}$ for $3 \mathrm{~min}$ and the resulting structure is shown in figures 6(d)-(f). During annealing, the diameter of the nanoparticles was reduced to about $30 \mathrm{~nm}$ and visible crystal facets/edges were formed. Some of the particles contained one stacking fault running through them, as can be seen in figure 6(e). The CBED pattern (figure 6(f)) could be indexed and shows that the Pt nanoparticles had been successfully transformed to single-crystal particles. There are weak spots visible in the diffraction pattern corresponding to forbidden reflections. They are most likely due to surface reconstruction of the (110) surface facets [35, 36]. Another possible explanation is a surface topography effect where parts of the particle have incomplete unit cells along the path of the incident electron beam disrupting the extinction of forbidden reflections [37]. However, this occurs for very small particles and, as these particles were about $30 \mathrm{~nm}$ in diameter, the effect of topography should be negligible.

By changing the atmosphere during annealing, it should be possible to control what facets are preferentially formed, since adsorbates change the relative surface energies of different facets [38]. Different atmospheres also affect the rate of annealing. In the specific case of $\mathrm{Pt}$ and $\mathrm{Pt}$ group metals oxygen is a well-known surfactant that speeds up annealing (and sintering) of Pt nanoparticles [3]. Being able to also control the crystallinity of the nanoparticles is of great value in the preparation of well-characterized nanostructured model systems both because it increases the reproducibility of the sample fabrication and especially as it enables mechanistic studies, where effects related to activity variations of different crystal facets and edges can be studied/accounted for.

\section{Conclusion}

We have demonstrated that electrodeposition can replace, or complement, evaporation as the means of producing the 
active material in hole-mask colloidal lithography on highly conductive $\mathrm{Au}$, as well as on less uniformly conductive amorphous $\mathrm{C}$ supports. The Pt nanodisc arrays displayed excellent mechanical and electrochemical stability and CV revealed that the sample surfaces were free from contaminants. The results are extendible to other lithographic techniques than CL. The demonstrated technique enables a broader use of lithography, as the need for complex equipment is reduced, which also lowers the cost associated with fabrication. It furthermore allows for fabrication of structures with higher aspect ratios than can be achieved with evaporation through a lithography mask, due to the fundamental differences in the growth mechanism between electrodeposition and evaporation of materials.

It was shown how nanoparticles with a much smaller size than the pattern defining colloidal particles can be produced by shrinking the holes in the hole-mask. This approach can also be used to prepare very sparse (large particle-particle distance) Pt arrays with preserved order, which is not possible with standard colloidal techniques.

Both evaporation and electrodeposition create polycrystalline films/particles, which were found to have a rather large roughness. Such variations and differences in morphology can complicate a comparison between samples prepared by different techniques, or at different conditions. By heattreating arrays of polycrystalline Pt nanoparticles it was shown that (and how) the particles can be transformed into single crystals. This enables mechanistic studies where crystal facets and edges, and the role of other defects, can be studied/accounted for. In summary, the techniques presented in this work may be used to prepare model systems with improved quality and precision, which can be applied to investigate electrochemical systems as well as gas phase catalytic studies.

\section{Acknowledgments}

The authors are grateful to Elisabet Ahlberg at the Department of Chemistry, University of Gothenburg for helpful tips regarding electrodeposition. Partial funding from the interconnected projects 'Nanotechnology for Sustainable Energy' from the Chalmers Foundation and 'Nano-SEE 181-1' from the Swedish Energy Agency is gratefully acknowledged. The work was partly performed at the Competence Centre for Catalysis, which is hosted by the Chalmers University of Technology and financially supported by the Swedish Energy Agency and the member companies: AB Volvo, Volvo Car Corporation, Scania CV AB, SAAB Automobile Powertrain Sweden AB, Haldor Topsøe A/S and the Swedish Space Corporation.

\section{References}

[1] Österlund L, Grant A and Kasemo B 2007 Nanocatalysis ed U Heiz and U Landman (Berlin: Springer) pp 269-341

[2] Somorjai G A and Park J Y 2008 J. Chem. Phys. 128182504

[3] Wong K, Johansson S and Kasemo B 1996 Faraday Discuss. $105237-46$

[4] Geissler M and Xia Y N 2004 Adv. Mater. 16 1249-69
[5] Deckman H W and Dunsmuir J H 1982 Appl. Phys. Lett. 41 377-9

[6] Yang S M, Jang S G, Choi D G, Kim S and Yu H K 2006 Small 2 458-75

[7] Hanarp P, Sutherland D S, Gold J and Kasemo B 2003 Colloids Surf. A $21423-36$

[8] Hulteen J C, Treichel D A, Smith M T, Duval M L, Jensen T R and Van Duyne R P 1999 J. Phys. Chem. B 103 3854-63

[9] Aizpurua J, Hanarp P, Sutherland D S, Käll M, Bryant G W and de Abajo F J G 2003 Phys. Rev. Lett. 90057401

[10] Kosiorek A, Kandulski W, Glaczynska H and Giersig M 2005 Small 1 439-44

[11] Lu Y, Liu G L, Kim J, Mejla Y X and Lee L P 2005 Nano Lett. 5 119-24

[12] Fredriksson H, Alaverdyan Y, Dmitriev A, Langhammer C, Sutherland D S, Zäch M and Kasemo B 2007 Adv. Mater. 19 4297-302

[13] Dmitriev A, Hägglund C, Chen S, Fredriksson H, Pakizeh T, Käll M and Sutherland D S 2008 Nano Lett. 8 3893-8

[14] Langhammer C, Kasemo B and Zoric I 2007 J. Chem. Phys. 126194702

[15] Larsson E M, Langhammer C, Zoric I and Kasemo B 2009 Science 326 1091-4

[16] Seidel Y E, Müller M, Jusys Z, Wickman B, Hanarp P, Kasemo B, Hörmann U, Kaiser U and Behm R J 2008 J. Electrochem. Soc. 155 K171-9

[17] Seidel Y E, Schneider A, Jusys Z, Wickman B, Kasemo B and Behm R J 2010 Langmuir 26 3569-78

[18] Seidel Y E, Lindström R W, Jusys Z, Gustavsson M, Hanarp P, Kasemo B, Minkow A, Fecht H J and Behm R J 2008 J. Electrochem. Soc. 155 K50-8

[19] Grant A W, Hu Q-H and Kasemo B 2004 Nanotechnology $151175-81$

[20] Klein M J K, Guillaumee M, Wenger B, Dunbar L A, Brugger J, Heinzelmann H and Pugin R 2010 Nanotechnology 21205301

[21] Plyasova L M, Molina I Y, Gavrilov A N, Cherepanova S V, Cherstiouk O V, Rudina N A, Savinova E R and Tsirlina G A 2006 Electrochim. Acta 51 4477-88

[22] Duarte M M E, Pilla A S, Sieben J M and Mayer C E 2006 Electrochem. Commun. 8 159-64

[23] Yasin H M, Denuault G and Pletcher D 2009 J. Electroanal. Chem. 633 327-32

[24] Biegler T, Rand D A J and Woods R 1971 J. Electroanal. Chem. 29 269-77

[25] Lindström R W, Seidel Y E, Jusys Z, Gustavsson M, Wickman B, Kasemo B and Behm R J 2010 J. Electroanal. Chem. 644 90-102

[26] Xu W, Wong J, Cheng C C, Johnson R and Scherer A 1995 J. Vac. Sci. Technol. B $132372-5$

[27] Ghanem M A, Bartlett P N, de Groot P and Zhukov A 2004 Electrochem. Commun. 6 447-53

[28] Bartlett P N, Baumberg J J, Birkin P R, Ghanem M A and Netti M C 2002 Chem. Mater. 14 2199-208

[29] Bartlett P N, Birkin P R and Ghanem M A 2000 Chem. Commun. 1671-2

[30] Hu J, Abdelsalam M, Bartlett P, Cole R, Sugawara Y, Baumberg J, Mahajan S and Denuault G 2009 J. Mater. Chem. 19 3855-8

[31] Despić A R and Jović V D 1995 Modern Aspects of Electrochemistry ed R E White (New York: Plenum) pp 143-232

[32] Lundquist J T and Stonehart P 1973 Electrochim. Acta 18 349-54

[33] Markovic N, Gasteiger H and Ross P N 1997 J. Electrochem. Soc. 144 1591-7

[34] Zhdanov V P and Kasemo B 1998 Surf. Sci. 405 27-37

[35] Conrad E H and Hörnis H 1995 Prog. Surf. Sci. 48 221-32

[36] Iddir H, Komanicky V, Ogut S, You H and Zapol P 2007 J. Phys. Chem. C 111 14782-9

[37] Krakow W 1979 Ultramicroscopy 4 55-76

[38] Zhdanov V P and Kasemo B 1998 Phys. Rev. Lett. 81 2482-5 